\title{
Forecast of the appearance of new strong sectors in the regional economy based on a probabilistic model
}

\author{
Mikhail Afanasiev*, and Aleksander Kudrov \\ Central Economics and Mathematics Institute of the Russian Academy of Sciences, Moscow, Russia
}

\begin{abstract}
The problem of forecasting the appearance of new strong sectors in the region shall be considered. Based on the methods of probabilistic and statistical modeling, a model has been built that makes it possible to assess the occurrence probability of a new strong sector in the region, taking into account the characteristics of the structure of the economy. The possibility of building such a model is based on the assumption that the appearance and development of sectors are largely due to the evolution of past economic activity. The model uses the indicators of nesting of structures of strong sectors of regional economies entered by the authors. These values are based on the probabilistic interpretation and properties of the matrix elements, which assesses the economic complexity, in accordance with the traditional approach. The condition for the appearance of a certain strong sector in the structure of the economy of a particular region with a probability exceeding 0.5 shall be obtained. This condition used to form a list of sectors recommended for priority development in the region. For each region in its structure, the occurrence probability of a specific sector as a strong one was estimated. On the basis of ordering the sectors by the value of these probabilities and assessing their potential contribution to socio-economic development, an expert assessment of the feasibility of developing a new strong sector in the region can be given. Research is focused on the development of theories of localized specialization and economic diversification.
\end{abstract}

\section{Introduction}

Let's define an indicator $R C A_{c p}$ of the revealed comparative advantages:

$$
R C A_{c p}=\frac{y_{c p / \Sigma_{p} y_{c p}}}{\sum_{c} y_{c p} / \Sigma_{c, p} y_{c p}}
$$

where $y_{c p}$ - the output of the sector $p$ of economy of the region $c$. Value $R C A_{c p}$ is the ratio of the share of production from the sector $p$ in the total volume of production from all sectors of the region's economy $c$ to the share of production of the sector $p$ for all regions in the volume of production from all sectors of the economy of all regions. According to the

\footnotetext{
*Corresponding author: mi.afan@yandex.ru
} 
work (Hausmann \& Klinger, 2006), to identify comparative advantages in economies, an indicator is used $R C A_{c p}$ for which a condition of the type of restriction from below is checked [7]:

$$
a_{c, p}=\left\{\begin{array}{l}
1, \text { if } R C A_{c p} \geq 1 \\
0, \text { if } R C A_{c p}<1
\end{array}\right.
$$

The matrix $A=\left(a_{c, p}\right)$ contains the data on economic sectors that are developed in various regions at the level of revealed comparative advantages, determined using expression (1). Lines of this matrix correspond to regions, columns - to sectors of economy. Let us call further a vector $\left(a_{c, p_{1}}, \ldots, a_{c, p_{m}}\right)$ the structure of strong sectors of economy of the regionc.

Let $R_{1}=\left(r_{c . p}\right)$ be a matrix with elements $r_{c . p}=\frac{a_{c . p}}{k_{c .0}}$, where $k_{c .0}=\sum_{p} a_{c . p}$.

Let $R_{2}=\left(r_{p, c}^{*}\right)$ be a matrix with elements $r_{p, c}^{*}=\frac{a_{c, p}}{k_{p, 0}}$, where $k_{p, 0}=\sum_{c} a_{c, p}$.

According to (Afanasiev \& Kudrov, 2020; Hausmann et al., 2011), the economic complexity of a region is defined as an eigenvector of the matrix $R_{1} R_{2}[1,6]$. Note that the element $w_{i, j}$ at the intersection of the $i$-th row and the $j$-th column of the matrix $R_{1} R_{2}$, that is $\left(R_{1} R_{2}\right)_{i j}$, is given by the formula:

$$
\frac{1}{k_{c_{i}, 0}} \sum_{t} \frac{a_{c_{i}, p_{t}} a_{c_{j}, p_{t}}}{k_{p_{t}, 0}}
$$

Let's note some properties of matrix $w_{i, j}$ elements:

1. Values $w_{i, 1}, \ldots, w_{i, n}$ may be interpreted as a probability distribution. The matrix $R_{1} R_{2}$ is stochastic, since the elements of the matrix are non-negative, and their row sum is 1 . Therefore, for each $i, j \in\{1, \ldots, n\}: w_{i, j} \geq 0, \sum_{j=1}^{m} w_{i, j}=1$.

2 . If a region $c_{i}$ holds one strong region, then $w_{i, i}>0$. Otherwise, $w_{i, i}=0$.

It is easy to show justice of this statement since:

$$
w_{i, i}=\frac{1}{k_{c_{i}, 0}} \sum_{t=1}^{m} \frac{a_{c_{i}, p_{t}}}{k_{p_{t}, 0}} \geq 0
$$

and the zero value is reached only when $a_{c_{i}, p_{t}}=0, t=1, \ldots, m$, but there are no such cases in our data.

3. Elements $w_{i, j}$ are equal to zero if and only if the condition is satisfied:

$$
\left\{t: a_{c_{i}, p_{t}}=1\right\} \cap\left\{t: a_{c_{j}, p_{t}}=1\right\}=\varnothing
$$

Performance of this condition means lack of the general strong sectors in structures of economy of regions $c_{i}$ and $c_{j}$.

4. In each row of the matrix $\left(w_{i, j}\right)$ the maximum element corresponds to the diagonal element, that is $w_{i, i}=\max _{j \in\{1, \ldots, n\}}\left(w_{i, j}\right)$. Let us show it.

Because of justice:

$$
w_{i, j}=\frac{1}{k_{c_{i}, 0}} \sum_{t=1}^{m} \frac{a_{c_{i}, p_{t}} a_{c_{j}, p_{t}}}{k_{p_{t}, 0}} \leq \frac{1}{k_{c_{i}, 0}} \sum_{t=1}^{m} \frac{a_{c_{i}, p_{t}}}{k_{p_{t}, 0}}=\frac{1}{k_{c_{i}, 0}} \sum_{t=1}^{m} \frac{a_{c_{i}, p_{t}} a_{c_{i}, p_{t}}}{k_{p_{t}, 0}}
$$


we receive that $w_{i, j} \leq w_{i, i}$. Moreover, equality in the last inequality is achieved only when the following condition is satisfied:

$$
\left\{t: a_{c_{i}, p_{t}}=1\right\} \subseteq\left\{t: a_{c_{j}, p_{t}}=1\right\}
$$

Fulfillment of this condition means that all strong sectors of the economic structure of the region $c_{i}$ are strong sectors in the structure of the region $c_{j}$ as well. If this condition is not met, then we have a strict inequality:

$$
\frac{w_{i, j}}{w_{i, i}}<1
$$

5. Asymmetry of a matrix $\left(w_{i, j}\right)$.

It is easy to show that $w_{j, i}=\frac{k_{c_{i}, 0}}{k_{c_{j}, 0}} w_{i, j}$. If the level of diversification of the region $c_{i}$ coincides with the level of diversification of the region $c_{j}$, then $w_{j, i}=w_{i, j}$; if the diversification of the region $c_{i}$ is lesser (higher) than the diversification of the region $c_{j}$, then $w_{j, i}>w_{i, j}\left(w_{j, i}<w_{i, j}\right)$. Thus, different levels of regional diversification guarantee the asymmetry of the matrix $\left(w_{i, j}\right)$.

From properties $(1-5)$ for $w_{i, j}$ it follows that the ratio $\frac{w_{i, j}}{w_{i, i}}$ can be interpreted as a characteristic of nesting level the region's set of strong sectors $c_{i}$ into the set of strong sectors for the region $c_{j}$. The lesser the ratio is, the lesser is the number of strong sectors of the region $c_{i}$ in the set $\left\{t: a_{c_{j}, p_{t}}=1\right\}$ of strong sectors of the region $c_{j}$.

The matrices $\left(a_{i, j}\right)$ and $\left(w_{i, j}\right)$, used below, as well as estimates of the economic complexity of the regions of the Russian Federation, calculated on the basis of data on tax revenues for 82 sectors of the economy, are presented in (Afanasyev, Kudrov, 2020) [1].

\section{Methods}

At present, algorithms for generating recommendations for selection shall be widely used [5]. A very general formulation of this task consists in recommending those choices that correspond to the greatest extent to the characteristics of the object for which the recommendations are being developed. For the structure of the strong sectors of the region's economy, the recommendations give a list of potentially achievable strong sectors given the characteristics of the region, as well as using information about strong sectors in similar regions.

Let the random variables

$$
A_{c_{i}, p_{k}}=\left\{\begin{array}{c}
1, \text { with probability } \pi\left(f_{c_{i}, p_{k}}^{1}, f_{c_{i}, p_{k}}^{2}, \ldots\right) \\
0, \text { with probability } 1-\pi\left(f_{c_{i}, p_{k}}^{1}, f_{c_{i}, p_{k}}^{2}, \ldots\right)
\end{array}\right.
$$

where $f_{c_{i}, p_{k}}^{1}, f_{c_{i}, p_{k}}^{2}, \ldots-$ are the factors influencing the occurrence of events $A_{c_{i}, p_{k}}=1$ and $A_{c_{i}, p_{k}}=0$. In what follows, we will assume that the dependence $\pi\left(f_{c_{i}, p_{k}}^{1}, f_{c_{i}, p_{k}}^{2}, \ldots\right)$ has the form:

$$
\pi\left(f_{c_{i}, p_{k}}^{1}, f_{c_{i}, p_{k}}^{2}, \ldots\right)=\frac{e^{\beta_{0}+\sum \beta_{h} f_{c_{i}, p_{k}}^{h}}}{1+e^{\beta_{0}+\sum \beta_{h} f_{c_{i}, p_{k}}^{h}}} \text { где } \beta_{0}-\text { constant }
$$


Suppose that the sequence $a_{c_{1}, p_{1}}, \ldots, a_{c_{1}, p_{m}}, \ldots, a_{c_{n}, p_{1}}, \ldots, a_{c_{n}, p_{m}}$ is a realization of a random sequence $A_{c_{1}, p_{1}}, \ldots, A_{c_{1}, p_{m}}, \ldots, A_{c_{n}, p_{1}}, \ldots, A_{c_{n}, p_{m}}$, the elements of which are assumed to be independent when $f_{c_{1}, p_{1}}^{1}, f_{c_{1}, p_{2}}^{1}, \ldots$ are given. In addition, suppose that distribution $P\left(A_{c_{i}, p_{k}} \mid f_{c_{1}, p_{1}}^{1}, f_{c_{1}, p_{2}}^{1}, \ldots\right)=P\left(A_{c_{i}, p_{k}} \mid f_{c_{i}, p_{k}}^{1}, \ldots, f_{c_{i}, p_{k}}^{h}, \ldots\right)$, that is, the factors influencing distribution $A_{c_{i}, p_{k}}$, are limited to the set $f_{c_{i}, p_{k}}^{1}, \ldots, f_{c_{i}, p_{k}}^{h}, \ldots$, which have the lower pair of indices $\left(c_{i}, p_{k}\right)$. Then the likelihood function has the form:

$$
\begin{gathered}
P\left(A_{c_{1}, p_{1}}=a_{c_{1}, p_{1}}, \ldots, A_{c_{1}, p_{m}}=a_{c_{1}, p_{m}}, \ldots, A_{c_{n}, p_{1}}=a_{c_{n}, p_{1}}, \ldots, A_{c_{n}, p_{m}}\right. \\
\left.=a_{c_{n}, p_{m}} \mid f_{c_{1}, p_{1}}^{1}, f_{c_{1}, p_{2}}^{1}, \ldots\right) \\
=P\left(A_{c_{1}, p_{1}}=a_{c_{1}, p_{1}} \mid f_{c_{1}, p_{1}}^{1}, \ldots, f_{c_{1}, p_{1}}^{h}, \ldots\right) \cdot \ldots \\
\cdot P\left(A_{c_{n}, p_{m}}=a_{c_{n}, p_{m}} \mid f_{c_{n}, p_{m}}^{1}, \ldots, f_{c_{n}, p_{m}}^{h}, \ldots\right) \\
=\frac{e^{a_{c_{1}, p_{1}}\left(\beta_{0}+\sum \beta_{h} f_{c_{1}, p_{1}}^{h}\right)}}{\left(1+e^{\left.\beta_{0}+\sum \beta_{h} f_{c_{1}, p_{1}}^{h}\right)}\right.} \cdot \ldots \cdot \frac{e^{a_{c_{n}, p_{m}}\left(\beta_{0}+\sum \beta_{h} f_{c_{n}, p_{m}}^{h}\right)}}{\left(1+e^{\left.\beta_{0}+\sum \beta_{h} f_{c_{n}, p_{m}}^{h}\right)}\right.}
\end{gathered}
$$

By maximizing this likelihood function, we obtain estimates of the parameters $\beta_{1}, \ldots, \beta_{h}, \ldots$. To assess the quality of the model, we will use the Akaike criterion, as well as the value:

$$
G=\frac{1}{m n} \sum_{i, k} I\left(I\left(\pi\left(f_{c_{i}, p_{k}}^{1}, f_{c_{i}, p_{k}}^{2}, \ldots\right)>0.5\right)=a_{c_{i}, p_{k}}\right)
$$

where $I(A)-$ is event indicator $A$. The value $G$ characterizes the proportion of cases correctly identified by the model.

Consider the ways of generating explanatory variables $f_{c_{i}, p_{k}}^{1}, \ldots, f_{c_{i}, p_{k}}^{h}, \ldots$

Method 1: for this method $h=2$. Let us define factors $f_{c_{i}, p_{k}}^{1} f_{c_{i}, p_{k}}^{2}$ for all $i=1, \ldots, n ; k=$ $1, \ldots, m$ :

$$
\begin{aligned}
f_{c_{i}, p_{k}}^{1} & =\frac{\sum_{j=1, i \neq j}^{n} w_{i, j} a_{c_{j}, p_{k}}}{1-w_{i, i}} \\
f_{c_{i}, p_{k}}^{2} & =\frac{\sum_{j=1, i \neq j}^{n} \frac{w_{j, i}}{w_{j, j}} a_{c_{j}, p_{k}}}{\sum_{j=1, i \neq j}^{n} \frac{w_{j, i}}{w_{j, j}}}
\end{aligned}
$$

Estimates of the parameters of the model (2)-(3) for this method of forming the explanatory factors are presented in Table 1 .

Table 1. Estimation of the parameters of the strong sector identification model for Method 1.

Source: (Compiled by the authors).

\begin{tabular}{|c|c|c|c|c|}
\cline { 2 - 5 } \multicolumn{1}{c|}{} & Estimate & Standarddeviation & t-statistics & p-value \\
\hline Constant & -2.94 & 0.07 & -43.42 & 0.00 \\
\hline$f_{c_{i}, p_{k}}^{1}$ & 2.91 & 0.83 & 3.52 & 0.00 \\
\hline$f_{c_{i}, p_{k}}^{2}$ & 18.15 & 0.91 & 20.12 & 0.00 \\
\hline \multicolumn{2}{|c|}{ G } & \multicolumn{2}{c|}{0.8} & \\
\cline { 2 - 4 } & AIC & \multicolumn{2}{c}{5681} & \multicolumn{1}{c}{} \\
\cline { 2 - 4 } & &
\end{tabular}


As can be seen from Table 1 , the coefficients at $f_{c_{i}, p_{k}}^{1}$ and $f_{c_{i}, p_{k}}^{2}$ are significant and positive. For this method of choosing explanatory variables, the proportion of cases correctly identified by the model is $80 \%$.

The simulation method was used to test the hypothesis that the index of nesting of the structure of strong sectors of the region $c_{i}, i=1, \ldots, n$, in the structure of strong sectors of the region $c_{j}, j=1, \ldots, n, j \neq i$ is equal to zero, namely:

$$
H_{0}: \frac{w_{i, j}}{w_{i, i}}=0 \text { contrary } H_{1}: \frac{w_{i, j}}{w_{i, i}} \neq 0
$$

It is shown that the 95\%-quantile for the distribution function $\frac{w_{i, j}}{w_{i, i}}$ under the conditions of validity of the null hypothesis is equal to 0.47 at a given level of significance $\alpha=0,95$.

Method 2: for this method $h=2$. Let us define factors $f_{c_{i}, p_{k}}^{1} f_{c_{i}, p_{k}}^{2}$ for all $i=1, \ldots, n ; k=$ $1, \ldots, m$ :

$$
\begin{gathered}
f_{c_{i}, p_{k}}^{1}=\frac{\sum_{j=1, i \neq j}^{n} I\left(\frac{w_{i, j}}{w_{i, i}}>0.47\right) w_{i, j} a_{c_{j}, p_{k}}}{\sum_{j=1, i \neq j}^{n} I\left(\frac{w_{i, j}}{w_{i, i}}>0.47\right) w_{i, j}} \\
f_{c_{i}, p_{k}}^{2}=\frac{\sum_{j=1, i \neq j}^{n} I\left(\frac{w_{j, i}}{w_{j, j}}>0.47\right) \frac{w_{j, i}}{w_{j, j}} a_{c_{j}, p_{k}}}{\sum_{j=1, i \neq j}^{n} I\left(\frac{w_{j, i}}{w_{j, j}}>0.47\right) \frac{w_{j, i}}{w_{j, j}}}
\end{gathered}
$$

Estimates of the parameters of the model (2)-(3) for this method of forming the explanatory factors are presented in Table 2 .

Table 2. Estimation of the parameters of the strong sector identification model for Method 2.

\begin{tabular}{|c|c|c|c|c|}
\cline { 2 - 5 } \multicolumn{1}{c|}{} & Assessment & $\begin{array}{c}\text { Standard } \\
\text { deviation }\end{array}$ & t-statistics & p-value \\
\hline Constant & -3.03 & 0.07 & -43.33 & 0.00 \\
\hline$f_{c_{i}, p_{k}}^{1}$ & 4.16 & 0.25 & 16.62 & 0.00 \\
\hline$f_{c_{i}, p_{k}}$ & 6.70 & 0.28 & 24.26 & 0.00 \\
\hline \multicolumn{2}{|c|}{$\mathrm{G}$} & 0.83 & \\
\cline { 2 - 4 } & $\mathrm{AIC}$ & \multicolumn{2}{c}{4950} \\
\cline { 2 - 4 }
\end{tabular}

Source: (Compiled by the authors).

As can be seen from Table 2, the coefficients at $f_{c_{i}, p_{k}}^{1}$ and $f_{c_{i}, p_{k}}^{2}$ are significant and positive. For this method of choosing the explanatory variables, the proportion of cases correctly identified by the model does not differ significantly from the proportion for case 2 and is equal to $83 \%$ of the total number of cases, but the value of the Akaike criterion is less than for the first method of choosing the explanatory variables. Method 2 is preferred.

\section{Results}

As a result of using the model presented above, lists of potentially strong sectors in the regions were formed. For example, for a number of regions specializing in agriculture, the development of the Transport and Storage sector is required, which makes it possible to have a bigger territorial coverage in the supply of manufactured products. Due to infrastructural problems, recommendations for the development of agriculture are also in the development of the sector 'Water intake, purification and distribution', which is necessary for the 
functioning of irrigation systems, as well as the sector 'Power supply, gas and steam; air conditioning', which is also required for the development of greenhouse complexes that require high energy consumption when creating a microclimate. It should also be noted that the development of agriculture contributes to the formation of demand for the development of the agricultural machinery sector, as well as many other sectors. As an example, Table 3 shows estimates of the occurrence probability of a sector 'Transportation and storage' in the regions as a strong sector. The range of identified potential strong sectors in the structures of regional economies based on the results of using the model is wider.

Table 3. Estimates of the occurrence probability of of the "transport and storage" sector as a strong.

\begin{tabular}{|c|c|}
\hline Region & Estimate of probability \\
\hline Belgorod region & 0.89 \\
\hline Voronezh region & 0.63 \\
\hline Kursk region & 0.57 \\
\hline Moscow region & 0.67 \\
\hline Saint Petersburg & 0.92 \\
\hline Republic of Adygea & 0.91 \\
\hline
\end{tabular}

\section{Discussions}

There are two main theories describing the mechanism of knowledge creation and distribution: localized specialization and economic diversification. Localized specialization theory was first presented in detail in the work (Marshall, 1890) and argues that companies surrounded by other representatives of the same industry will grow faster due to the circulation of knowledge within the industry [9]. This theory was further developed in the works (Arrow, 1962; Romer, 1986) [2,11]. The opposite theory and the resulting empirical estimates are presented in (Blien \& Wolf, 2006; Fuchs, 2011; Illy, Schwartz, Hornych \& Rosenfeld, 2011). According to this theory, companies benefit from the fact that they are faced with a heterogeneous environment, consisting of different industries, as new ideas come from the external environment $[3,4,8]$. Variety leads to economic growth through mechanisms called diversification. Researches on theories of localization or diversification suggest that a choice shall be made in favor of one or another.

The results obtained do not contradict the assumption that the effects of localization and diversification can complement each other and are not mutually exclusive. Estimates of the occurrence probability of a specific sector in the structure of the region as a strong one indicate that the appearence and development of sectors is largely due to the evolution of past economic activity. These results are consistent with the conclusions in the work (Neffke, Henning \& Boschma, 2011), which shown that it is easier for regions to develop new industries if they are associated with already existing ones in the region [10].

\section{Conclusion}

A probabilistic interpretation of the elements of the matrix by which the economic complexity shall bes estimated from the work (Hausmann et al., 2011) is shown. Their properties are given, on the basis of which indicators are introduced that characterize the nesting of the structures of strong sectors of regional economies [6].

A model has been built that explains the appearance and absence of a strong sector in the structure of the region's economy. On this basis, the condition for the appearance of a certain strong sector in the structure of the economy of a particular region with a probability 
exceeding 0.5 shall be obtained. This condition used to form a list of sectors recommended for priority development in the region. On the basis of ordering the sectors by the value of these probabilities and assessing their potential contribution to socio-economic development, an expert assessment of the feasibility of developing a new strong sector in the region can be given.

\section{References}

1. M. Yu. Afanasiev, A.V. Kudrov, Montenegrin Journal of Economics 16(4), 43 (2020)

2. K. J. Arrow, The Review of Economic Studies 29(3), 155 (1962)

3. U. Blien, K. Wolf, Labour Economics 13(4), 445 (2006)

4. M. Fuchs, Empirical Economics 40(1), 177 (2011)

5. K. Glodberg, T. Roeder, D. Gupta, C. Perkins, Information Retrieval 4, 133 (2001)

6. R. Hausmann, C. Hidalgo, S. Bustos, M. Coscia, A. Simoes, M. A. Yildirim, The Atlas of Economic Complexity. Mapping Paths to Prosperity. Cambridge, MA: Center for International Development, Harvard University, Harvard Kennedy School, Macro Connections, Massachusetts Institute of Technology (2011)

7. R. Hausmann, B. Klinger, SSRN Electronic Journal (2006)

8. A. Illy, M. Schwartz, C. Hornych, M. Rosenfeld, Journal of Economic and Social Geography 102(5), 582 (2011)

9. A. Marshall, Principles of Economics (London, UK: MacMillan, 1890)

10. F. Neffke, M. Henning, R. Boschma, Economic Geography. 87(3), 237 (2011)

11. P. M. Romer, Journal of Political Economy 94(5), 1002 (1986) 